\title{
The effect of Ramadan fasting on spatial attention through emotional stimuli
}

This article was published in the following Dove Press journal:

Psychology Research and Behavior Management

24 May 2016

Number of times this article has been viewed

\author{
Maziyar Molavi \\ Jasmy Yunus \\ Nugraha P Utama \\ Department of Clinical Sciences, \\ Faculty of Biosciences and Medical \\ Engineering (FBME), Universiti \\ Teknologi Malaysia (UTM), Johor \\ Bahru, Johor, Malaysia
}

\begin{abstract}
Fasting can influence psychological and mental states. In the current study, the effect of periodical fasting on the process of emotion through gazed facial expression as a realistic multisource of social information was investigated for the first time. The dynamic cue-target task was applied via behavior and event-related potential measurements for 40 participants to reveal the temporal and spatial brain activities - before, during, and after fasting periods. The significance of fasting included several effects. The amplitude of the N1 component decreased over the centroparietal scalp during fasting. Furthermore, the reaction time during the fasting period decreased. The self-measurement of deficit arousal as well as the mood increased during the fasting period. There was a significant contralateral alteration of $\mathrm{P} 1$ over occipital area for the happy facial expression stimuli. The significant effect of gazed expression and its interaction with the emotional stimuli was indicated by the amplitude of N1. Furthermore, the findings of the study approved the validity effect as a congruency between gaze and target position, as indicated by the increment of P3 amplitude over centroparietal area as well as slower reaction time from behavioral response data during incongruency or invalid condition between gaze and target position compared with those during valid condition. Results of this study proved that attention to facial expression stimuli as a kind of communicative social signal was affected by fasting. Also, fasting improved the mood of practitioners. Moreover, findings from the behavioral and event-related potential data analyses indicated that the neural dynamics of facial emotion are processed faster than that of gazing, as the participants tended to react faster and prefer to relay on the type of facial emotions than to gaze direction while doing the task. Because of happy facial expression stimuli, right hemisphere activation was more than that of the left hemisphere. It indicated the consistency of the emotional lateralization concept rather than the valence concept of emotional processing.
\end{abstract}

Keywords: fasting, emotion, spatial attention, ERP, hemisphere lateralization

\section{Introduction}

Fasting is a practice where nothing is taken into the digestive system for a certain period of time, and this is practiced by many people globally, either under cultural/ traditional or religious contexts. Many people believe that fasting enhances the mental and spiritual alertness of the practitioners. The Islamic community has a special month, named Ramadan, to practice fasting, when they restrain from intake of any food and water from dawn till dusk throughout the month.

Several previous studies have reported that fasting is frequently accompanied by a subjective feeling of well-being and a mood improvement. ${ }^{1-3}$ As a consequence, the mood enhancement contributes to successful social interactions and a longer life span. Previous research declared that a positive mood broadens the scope of spatiotemporal
Correspondence: Nugraha P Utama Department of Clinical Sciences, Faculty of Biosciences and Medical Engineering, Universiti Teknologi Malaysia (UTM), 81310 Johor Bahru, Johor, Malaysia Tel +6075558506

Fax +60755585I5

Email utama@biomedical.utm.my
Psychology Research and Behavior Management 2016:9 I05-II4

Dovepress

http://dx.doi.org/10.2147/PRBM.SI00495 (c) (i) (5) 2016 Molavi et al. This work is published and licensed by Dove Medical Press Limited. The full terms of this license are available at https://www.dovepress.com/terms. cC. you hereby accept the Terms. Non-commercial uses of the work are permitted without any further permission from Dove Medical Press Limited, provided the work is properly attributed. For permission for commercial use of this work, please see paragraphs 4.2 and 5 of our Terms (https://www.dovepress.com/terms.php). 
attention, provides more flexibility, and biases the attention to positive stimuli. ${ }^{4-6}$

The main aim of this study was to evaluate the effect of periodic Ramadan fasting on attention to dynamic spatial emotion stimuli. To provide for more realistic experiments, we used the gazed facial expression through the dynamic cue-target paradigm.

Social referencing is a spatial term of developmental psychology that explains the role of emotional expression and gazed cue as a powerful nonverbal social signal. This ability in detecting nonverbal social signal was believed to be an ontogenetic process that started from early pregnancy.

The effect of interaction between emotional type and gaze direction still remains unclear. Some previous studies have stated that there were positive interactions between emotional type and gaze direction, ${ }^{7,8}$ but other studies have stated that they found no interaction between emotional type and gaze direction. ${ }^{9,10}$ To elucidate the interaction between emotional type and gaze direction, this study evaluates the dynamics of neural activities using event-related potential (ERP) analysis in correlation with behavioral data. Previous research has considered the significant change of attention through behavioral and ERP components when it is involved between fearful and neutral faces associated with the withdrawal-oriented term. ${ }^{11}$ On the other hand, with the aforementioned findings of the previous studies, the valence theory indicates that positive emotion through the approach-oriented mechanism produces a broadening of spatial attention, thereby recruiting more resources from the left hemisphere. ${ }^{6,12,13}$

Contrary to the valence theory, the lateralized theory described that all types of emotional stimuli were processed in the right hemisphere only. ${ }^{14-20}$ Our study was the first among others that focused on happy facial expression in comparison with neutral expression to explore the spatiotemporal aspect of the neural process through ERPs and behavioral results. To provide more realistic emotional signals, the dynamic cue-target method was used as suggestion by Friesen and Kingstone. ${ }^{21}$

\section{Materials and methods Participants}

A total of 40 members (20 males and 20 females with an age range of $26 \pm 3.1$ years) who were postgraduate students of Universiti Teknologi Malaysia (UTM) participated voluntarily in this study. Data collection from three periods before, during, and after the fasting period of Ramadan - was done for every participant. Some of the participants (one before fasting period, and one during fasting period) did not attend the test. No participants were reported to have neurological and psychiatric illness during the interview prior to experiment, and they were mentally and physically fit to practice the fasting. All participants had normal or corrected-to-normal visual acuity. All participants had signed the written consent and agreement that had been approved by the research ethics committee of UTM in accordance with the Helsinki protocol, prior to joining the experiment.

\section{Stimulus}

Stimuli were taken from the collection of Radboud Faces Database, ${ }^{22}$ where face images of happy and neutral emotional classes of one man-model (\#090-015) were adopted. Each emotional class comprised a straight face, left-gazed face, and right-gazed face. To reduce any confounding variables in ERP analysis, this study took face images from only one model (Figure 1).

\section{Task parameters}

To elucidate the effect of fasting on attention, the test and data collection were repeated three times - within 1 month before the Ramadan, within 1 month during the Ramadan, and within 1 month after the Ramadan. To see the effect of periodic fasting, each subject should have spent at least 1 week practicing the islamic Ramadan fasting before the data collection, and for the nonfasting effect, participants should have not fasted at least 1 week before the data collection.

In every period of data collection, each participant performed the experiment in four runs, in which each trial continued for almost 4.4 minutes. Every run included 92 trials, which consisted of 8 repetitions of 8 target trials and 7 repetitions of 4 nontarget trials (catch trials). The dynamic cue target started with a neutral straight face in the middle of the screen for 300 ms. Following the neutral face image, a semifacial expression was presented for $50 \mathrm{~ms}$ to onset the gazing and emotional expressions simultaneously. This semifacial image was made using Winmorph 3.0.1 (WinMorph, Sibiu, Romania) and Adobe Photoshop 5.5 (Adobe, Mountain View, CA, USA). ${ }^{23}$ Four digitized gray-scale photographs were used in this section. These images constituted the factorial combination of facial expression (happy and neutral) and gazed direction (left and right). To generate realistic changes in emotional expression, the happy facial expressions of intermediate intensity were created by utilizing the morphing methods outlined by LaBar et $\mathrm{al}^{23}$ using Winmorph 3.0.1 software. The morphs depicting 55\% happy and 45\% neutral expressions were created with the left and right gaze. Furthermore, they were utilized to create a more naturalistic apparent motion effect. 


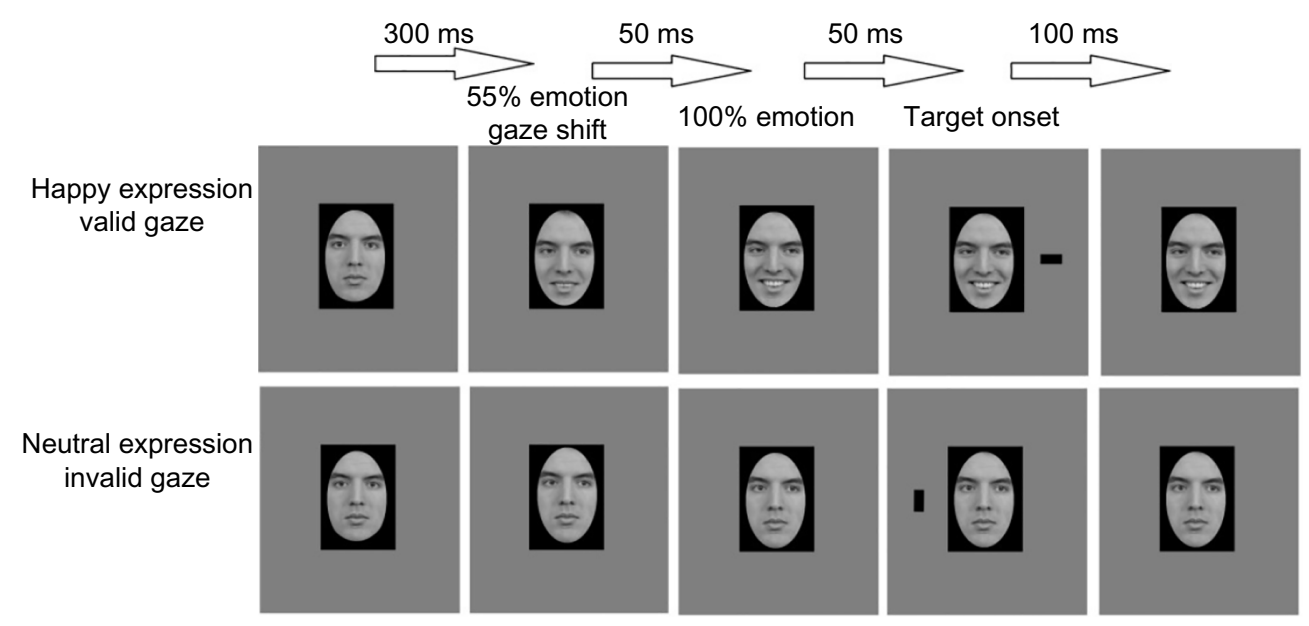

Figure I The experimental design.

Notes: Neutral facial stimulus was presented for $300 \mathrm{~ms}$ followed by happy or neutral facial expression. For happy facial expression stimuli, there were two stages of emotional level; $55 \%$ and $100 \%$ of emotional level, each of which was presented consecutively for 50 ms (upper row). For neutral facial expression, the same neutral expression was presented for last $100 \mathrm{~ms}$ (lower row). The gaze shift was started from $300 \mathrm{~ms}$ after stimuli onset for both stimuli of happy and neutral facial expression. The target was presented for $100 \mathrm{~ms}$ where valid meant the gaze is onto target location, and invalid meant the gaze is out to target location. Participants needed to respond during the period of I,000 ms after target onset. Whole trial duration was maximally I,400 ms with a random intertrial interval between 500 and I,000 ms.

After semifacial face presentation, fully emotional or neutral face with eye-gaze direction was presented for $50 \mathrm{~ms}$, followed by target presentation in the left or right side of visual field for $100 \mathrm{~ms}$ with the same face image. Participants were asked to press certain buttons within 1,000 ms after the target onset. The task was to discriminate between two targets - vertical-rectangular and horizontal-rectangular. There was time interval of fixation point in the range of $500-1,000$ ms between trials.

In this experiment, we used the terms "valid" and "invalid" for indicating the congruency between target and eye-gaze direction, where valid trial meant target appeared in the same direction as eye-gaze and invalid trial meant target appeared in the opposite direction of eye-gaze. The target was presented with a vertical visual angle of $2.15^{\circ}$ above the center of display, and a horizontal visual angle of $7.45^{\circ}$ to the right or left of the center of the display. Facial images were displayed at the center of the display with a horizontal visual angle of $6.5^{\circ}$ and a vertical visual angle of $8.7^{\circ}$.

The vertical- or horizontal-rectangular targets, which were applied in the left or right side, were placed at the same rate. The targets were subtended $1.1^{\circ} \times 1.6^{\circ}$ of the visual angle. Furthermore, the cue stimuli were applied randomly as leftward gaze versus rightward gaze and happy versus neutral expression at the same rate. In each run, about onethird of the trials presented as nontarget trial (catch trial) tasks to eliminate the effects of brain activity during the cue processing. ${ }^{24}$

\section{Behavioral data}

The mood and deficit arousal manipulation were evaluated in this study by distributing a questionnaire, which was developed by Becker and Leinenger. ${ }^{25}$ The questionnaire consisted of four questions for measuring tiredness, tension, happiness, and pleasantness level. Deficit arousal was the average of tiredness and tension level. Also, the mood level was the average of happiness and pleasantness level.

The results were analyzed by repeated measures analysis of variance (ANOVA) using SPSS version 18 (SPSS Inc., Chicago, IL, USA). In addition, reaction time (RT) was analyzed by repeated measures ANOVA within four factors: (fasting, not-fasting $) \times($ facial emotion: happy, neutral $) \times$ (validity: valid trial, invalid trial) $\times$ (cue gaze: left gazed, right gazed).

\section{Event-related potential}

The data acquisition was carried out with an electroencephalograph (EEG-9100 nihone kohden, Nihone Kohden, Nakano, Tokyo) with a 19-channel standard electrode cap with $\mathrm{Ag} / \mathrm{AgCl}$ electrodes that combined with two bipolar electrodes. Throughout the data collection, all the electrodes' impedance was kept constant, and below $5 \mathrm{k} \Omega$ for all scalp electrodes and below $10 \mathrm{k} \Omega$ for the electrooculograph (EOG). The EOG for each electrode was digitized at $1,000 \mathrm{~Hz}$ and analyzed with Eeglab version 13.4.4b and Erplab version 4.0.3.1. The target trials were categorized into eight bins: 2 cue emotions $\times 2$ cue gazed $\times 2$ target position. Also, the catch trials were divided into four bins: 2 cue emotions $\times 2$ cue gazed. 
The original brain waves were filtered using band-passed filter $(0.01-45 \mathrm{~Hz})$ and epoched in the range of -200 to $800 \mathrm{~ms}$ based on target onset, where data of -200 to $0 \mathrm{~ms}$ were set as baseline. Manual eye inspection with automatic rejection for amplitude exceeding $50 \mu \mathrm{V}$ was applied to the epoched data, which rejected about $6 \%$ of the total data.

The epoched data after artifact rejection were considered to be clean data for further analyses. Based on a previous study, ${ }^{11}$ we focused our analyses on three components of ERP P1 (115-145 ms), N1 (175-205 ms), and P3 (300-350 ms). Data from these three ERP components were then analyzed by repeated measures ANOVA within four factors - (fasting, not-fasting $) \times($ facial emotion: happy, neutral $) \times($ validity: valid, invalid $) \times$ (gazing: left, right $)-$ in order to reveal the effect of experimental variables.

\section{Results}

To elucidate the effects of fasting, two comparisons were conducted - before fasting versus during fasting, and after fasting versus during fasting. As explained earlier, before fasting data were those that were taken before the month of Ramadan, during fasting data were those that were taken in the month of Ramadan, and after fasting data were those that were taken after the month of Ramadan. From here on, we designated before fasting versus during fasting as step 1 and after fasting versus during fasting as step 2 .

Step 1 comprised data from 38 participants to be evaluated and compared, whereas step 2 comprised data from 39 participants.

Based on the mood analysis and deficit arousal selfreporting, the findings of the study indicated that the deficit arousal has two subcomponents, which are tiredness and tension. For those two subcomponents, only subcomponent of tiredness was increased under the fasting condition in both the steps (Table 1). The results suggested that the increment of deficit arousal was attributed to the increase of tiredness during the fasting period.

The level of mood was taken as the mean of happiness and pleasantness level. Furthermore, the result also indicated that happiness and pleasantness were increased during fasting, compared with either before or after fasting. In addition, repeated measures ANOVA indicated that the amount of mood level was significantly increased during fasting compared with that before and after fasting.

\section{Reaction time}

A four-way repeated measures ANOVA through $2 \times 2 \times 2 \times 2$ factors - (fasting, not-fasting) $\times$ (facial emotion: happy, neutral $) \times($ validity: valid, invalid $) \times($ gazing: left, right $)-$ reported the main significant effects on fasting, emotion, validity, and gaze variables in both steps, respectively (Table 2). Hence, the results of the dynamic emotional test indicated that the fasting RT was significantly slower than that in the nonfasting period. In addition, the mean RT, which included happy facial image stimuli, was significantly faster than that of trials that included neutral facial image stimuli.

Regarding the validity effect, the results of the study demonstrated that the validity effect was more significant and faster in a valid condition. Another significant effect that could be analyzed in this study was related to the gazed cue. The result indicated that left-gazed cue trial had faster RT compared with that of the right-gazed cue. In this study, we found that target position had no significant effect in both step 1 and step 2.

Four definitions - difference of reaction time (dRT), difference of emotional reaction time (dERT), difference of deficit arousal (dDA), and difference of mood factor $(\mathrm{dDM})$ - were introduced in this study. These four definitions represented the differences of related parameters between the fasting and nonfasting conditions.

Table I Effect of fasting on self-measurement components

\begin{tabular}{|c|c|c|c|c|c|c|c|c|}
\hline \multirow[t]{2}{*}{$\begin{array}{l}\text { Self-measurement } \\
\text { subcomponents }\end{array}$} & \multicolumn{4}{|c|}{$\begin{array}{l}\text { Step I: comparison between before fasting and } \\
\text { during fasting for } 38 \text { persons }\end{array}$} & \multicolumn{4}{|c|}{$\begin{array}{l}\text { Step 2: comparison between during fasting and } \\
\text { after fasting for } 39 \text { persons }\end{array}$} \\
\hline & $\begin{array}{l}\text { Before fasting } \\
\text { (mean level) }\end{array}$ & $\begin{array}{l}\text { During fasting } \\
\text { (mean level) }\end{array}$ & Sig & $F$ & $\begin{array}{l}\text { During fasting } \\
\text { (mean level) }\end{array}$ & $\begin{array}{l}\text { After fasting } \\
\text { (mean level) }\end{array}$ & Sig & $F$ \\
\hline (A) Deficit arousal & 3.62 & 6.62 & $P<0.0$ I & 141.3 & 6.51 & 3.65 & $P<0.0$ I & 99.31 \\
\hline (AI) Tiredness & 2.01 & 8.03 & $P<0.0$ I & 94.8 & 8.12 & 2.11 & $P<0.0$ I & 91.03 \\
\hline (A2) Tension & 5.23 & 5.18 & $P=0.8$ & 0.07 & 5.21 & 5.27 & $P=0.7$ & 0.08 \\
\hline (B) Mood & 4.30 & 6.023 & $P<0.01$ & 66.02 & 4.31 & 6.13 & $P<0.01$ & 25.85 \\
\hline (BI) Happiness & 4.33 & 5.91 & $P<0.01$ & 41.60 & 5.82 & 4.80 & $P=0.01$ & 13.79 \\
\hline (B2) Pleasantness & 4.41 & 6.11 & $P<0.01$ & 48.96 & 6.21 & 4.53 & $P<0.01$ & 43.44 \\
\hline
\end{tabular}

Abbreviations: sig, significant; F, F-test of repeated measures ANOVA; ANOVA, analysis of variance. 
Table 2 Reaction time results using the repeated measures ANOVA

\begin{tabular}{|c|c|c|c|c|c|c|c|}
\hline \multirow[t]{2}{*}{ Factor } & \multirow[t]{2}{*}{ Condition } & \multicolumn{3}{|c|}{$\begin{array}{l}\text { Step I: comparison between before } \\
\text { fasting and during fasting for } 38 \text { persons }\end{array}$} & \multicolumn{3}{|c|}{$\begin{array}{l}\text { Step 2: comparison between during } \\
\text { fasting and after fasting for } 39 \text { persons }\end{array}$} \\
\hline & & $\begin{array}{l}\text { Mean reaction } \\
\text { time }(\mathrm{ms})\end{array}$ & Sig & $F_{1,37}$ & $\begin{array}{l}\text { Mean reaction } \\
\text { time }(\mathrm{ms})\end{array}$ & Sig & $F_{1,38}$ \\
\hline \multirow[t]{2}{*}{ Fasting } & Fasting & 414 & $P<0.001$ & 149.68 & 410 & $P<0.001$ & 192.39 \\
\hline & Not-fasting & 393 & & & 397 & & \\
\hline \multirow[t]{2}{*}{ Facial emotion } & Happy & 398 & $P<0.001$ & 140.17 & 397 & $P<0.001$ & 192.39 \\
\hline & Neutral & 411 & & & 410 & & \\
\hline \multirow[t]{2}{*}{ Gaze } & Left & 399 & $P<0.001$ & 219.22 & 389 & $P<0.001$ & 98.01 \\
\hline & Right & 408 & & & 398 & & \\
\hline \multirow[t]{2}{*}{ Validity } & Valid & 402 & $P=0.037$ & 4.66 & 402 & $P<0.001$ & 55.24 \\
\hline & Invalid & 405 & & & 407 & & \\
\hline
\end{tabular}

Abbreviations: sig, significant; $F, F$-test of repeated measures ANOVA; ANOVA, analysis of variance.

The correlation between dRT and dDA can be identified using Spearman's rank correlation coefficient (Spearman's $\rho$ ) and Pearson product-moment correlation coefficient (Pearson's $r$ ). The result of the correlation between dRT and dDA showed significant correlation as follows: (step 1: Spearman's $\rho:[r=0.725, P<0.01]$; Pearson's $r:[r=0.946$, $P<0.01]$; step-2: Spearman's $\rho:[r=0.886, P<0.01]$; Pearson's $r:[r=0.978, P<0.01])$. The result also specified that $\mathrm{dDM}$ and $\mathrm{dERT}$ were significantly correlated in both steps through both methods: (step 1: Spearman's $\rho$ : $[r=0.820$, $P<0.01]$; Pearson's $r$ : $r=0.899, P<0.01]$; step 2: Spearman's $\rho:[r=0.875, P<0.01]$; Pearson's $r:[r=0.970, P<0.01])$.

\section{Event-related potential}

EEG data of doing experimental task before, during, and after Ramadan were analyzed for ERP analysis, where the onset of target appearance was set as the onset of ERP analysis.

By referring to previous research,, ${ }^{911}$ we focused on the first three important ERP components, which were P1 (115-145 ms), N1 (175-205 ms), and P3 (300-350 ms). Based on the result, P1 was activated more over parietooccipital electrodes (O1 and $\mathrm{O} 2)$, whereas $\mathrm{N} 1$ and $\mathrm{P} 3$ were activated more over centroparietal electrodes $(\mathrm{Cz}$ and $\mathrm{Pz})$ (Figure 2).

The ERP analyses on the factors of fasting (fasting, nonfasting), emotional cue (neutral, happy), gaze direction (left, right), and validity (valid, invalid) were conducted by the repeated measures ANOVA in the step 1 (before fasting, during fasting) and step 2 (during fasting, after fasting).

For the effect of emotional cue in brain activity, P1 showed greater amplitude for happy-image stimuli compared with that for neutral image stimuli. The position of target affected the P1-amplitude contralateral; P1-amplitude was greater when the target appeared at the contralateral visual view. $\mathrm{P} 1$-amplitude at $\mathrm{O} 1$ was greater when the target was on the right-visual view rather than on the left-visual view, whereas at $\mathrm{O} 2$, the reverse was true (Figure 3). (O1: step-1: $\mathrm{F}_{1,37}=50.07, \mathrm{P}<0.01 ;$ step- $2: \mathrm{F}_{1,38}=68.59, \mathrm{P}<0.01 ; \mathrm{O} 2$ : step$1: \mathrm{F}_{1,37}=113.45, \mathrm{P}<0.01$; step-2: $\left.\mathrm{F}_{1,38}=176.65, \mathrm{P}<0.01\right)$.

For the effect of fasting and nonfasting factors in brain activity, N1 showed greater amplitude for the fasting period compared with that for the nonfasting period. This effect happened significantly over $\mathrm{Cz}$ and $\mathrm{Pz}$ electrodes in both steps (Figure 4). (Cz: step-1: $F_{1,37}=36.324, P<0.01$; step-2: $F_{1,38}$ $=50.98, P<0.01$; Pz: step-1: $F_{1,37}=68.26, P<0.01$; step-2: $\left.F_{1,38}=88.44, P<0.01\right)$.

For the gaze cue effect, the N1-amplitude exposed greater significant value for rightward gazing over $\mathrm{Cz}$ and Pz electrodes in both steps compared with that for leftward gazing (Cz: step 1: $F_{1,37}=369, P<0.01$; step 2: $F_{1,38}=225.85$, $P<0.01$; Pz: step-1: $F_{1,37}=311.22, P<0.01$; step-2: $F_{1,38}$ $=264.7, P<0.01)$. Post hoc analyses using Bonferroni correction revealed interesting results with regard to the interaction between gaze cue and emotional cue. The results indicated that rightward gaze cue accompanied by happy face stimuli induced significantly greater N1-amplitude than that accompanied by neutral face image (Cz: step 1: $P<0.01$; step 2: $P<0.01$; Pz: step 1: $P<0.01$; step 2: $P<0.01$ ). However, post hoc analyses using Bonferroni correction showed this trend was reversed for leftward gaze. The leftward gaze cue accompanied by neutral face stimuli induced significantly greater N1-amplitude than that accompanied by happy face image (Cz: step 1: $P<0.01$; step 2: $P<0.01$; Pz: step 1: $P<0.01$; step 2: $P<0.01$ ) (Figure 5).

For the effect of validity in brain activity, the $\mathrm{P} 3$ component showed greater amplitude for invalid trials compared with that for valid trials. This phenomenon could be found at $\mathrm{Cz}$ and $\mathrm{Pz}$ electrodes for both steps (Cz: step 1: $F_{1,37}$ 


\section{A P1 component}

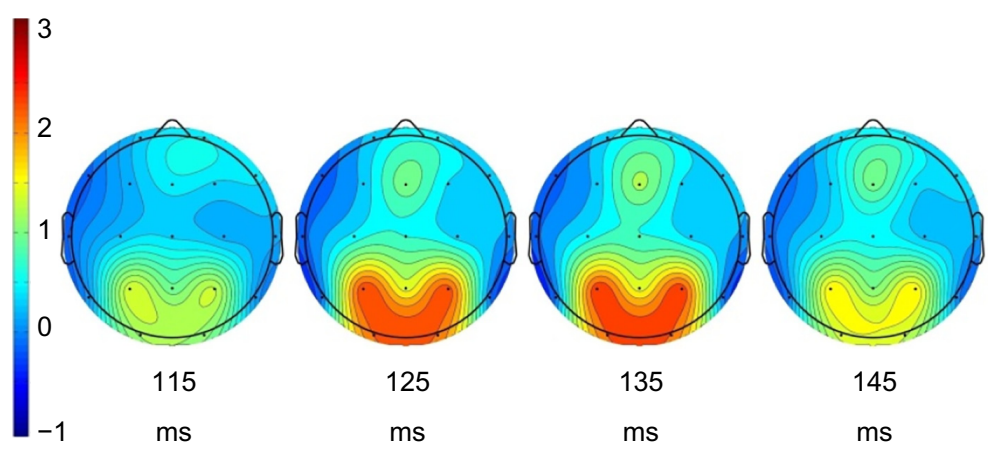

$\mu \nu$

\section{B N1 component}
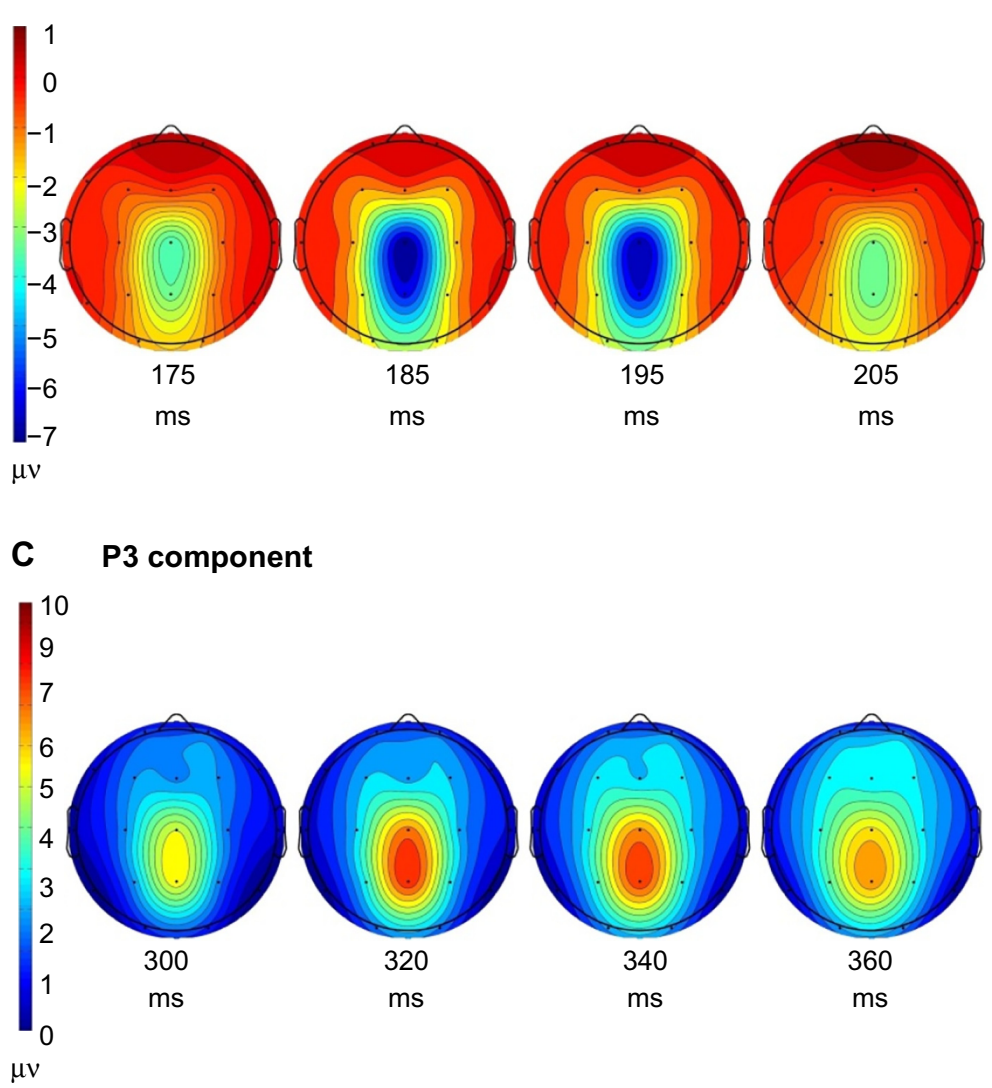

Figure 2 The scalp topography at the peak of three components.

Notes: (A) More activation of PI component (II5-145 ms) over parieto-occipital electrodes (OI and O2). (B) More activation of NI component (I75-205 ms) over centroparietal electrodes ( $\mathrm{Cz}$ and Pz electrodes). (C) More activation of P3 component (300-350 ms) over centroparietal electrodes (Cz and Pz electrodes).

$=80.01, P<0.01 ;$ step 2: $F_{1,38}=69.05, P<0.01 ;$ Pz: step 1: $F_{1,37}=96.032, P<0.01 ;$ step $\left.2: F_{1,38}=85.924, P<0.01\right)$ (Figure 6).

\section{Discussion}

Many previous studies explored facial expression and gaze cue as the parameters of nonverbal communication, ${ }^{26-29}$ but only a few of them had tried to study the interaction among these three parameters - emotional cue, gaze cue, and fasting. Fasting is believed by the practitioners to have an effect in their social communication.

The main objective of this study was to elucidate the effect of fasting and its interaction with facial expression and gaze cue in nonverbal communication by analyzing the ERP features. Unlike previous studies that used dynamic face as the dynamic faces as stimuli, ${ }^{9-11}$ we directly compared the 
A

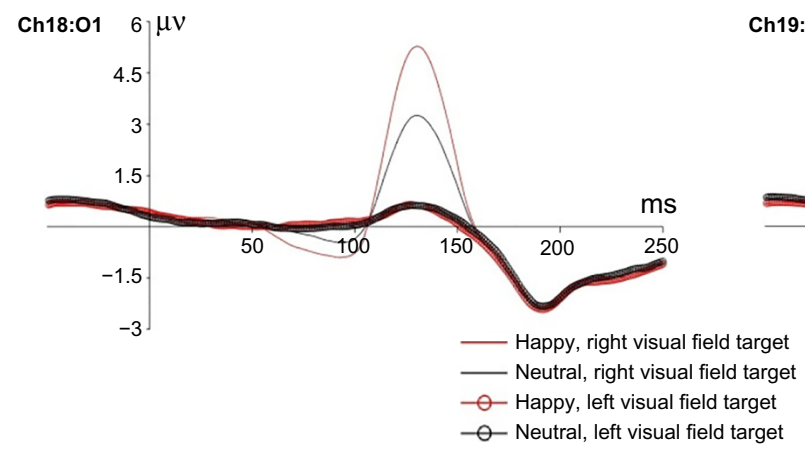

B

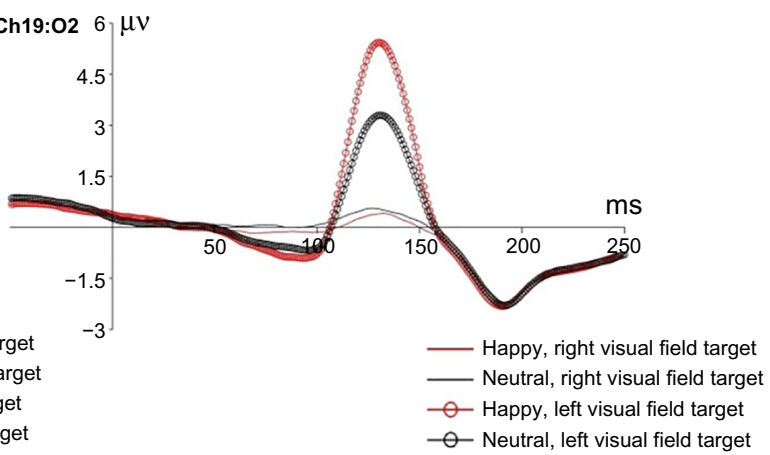

Figure 3 The effect of emotional facial stimuli on the PI component to left- and right-visual targets.

Notes: (A) The effect of left- and right-visual targets over left hemisphere (OI). (B) The effect of left- and right-visual targets over right hemisphere (O2). The figure shows that the PI over electrodes in contralateral hemisphere to visual target has bigger amplitude in happy facial stimuli than that in neutral facial stimuli.

emotional face image with neutral face image to elucidate the effect of emotional cue across the ERP components and behavioral data. By comparing the ERP components directly with regard to emotional cue, we were able to get new insights about the neural dynamics of emotional perception in nonverbal communication.

On the basis of the behavioral results, we infer that happy facial image stimuli helped the participants in selecting the target faster, indicated by the decrement of RT, compared with that with neutral facial image stimuli. This decrement in RT was indicated by the increment of P1-amplitude because of happy facial stimuli rather than that because of neutral facial stimuli. This increment of P1-amplitude could be explained as a preprocessing of visual objects in extrastriate visual cortex. ${ }^{30,31}$ These findings suggest that the brain started to differentiate the facial emotion in extrastriate visual cortex prior to full recognition of the facial emotion. This differentiation of facial emotion might alert participants to respond quicker, which is indicated by the decrement of RT compared with that of neutral facial emotion. This result was the same as that in a previous behavioral study, which suggested that the integration of facial emotion and gaze direction under tensed condition was implicated to human behavioral output, either to approach or to avoid. ${ }^{26}$ Actually, close association between the emotional valence and the approach avoidance has been covered by the evidence that approach motivation is triggered by positive emotional stimulus and avoidance motivation is triggered by negative emotional inputs. ${ }^{32}$ The shifting from neutral state to happy facial stimuli (approached state) may cause to engage more and prior neural resource to biased attention.

Although the effect of emotional factor influenced the P1 component as an early processing feature, the effect of gaze factor did not elicit this component. Therefore, these results suggested that during social behavior with faster and individual dynamics, the facial emotion was more crucial as a decisive cue rather than the gaze direction. However, some previous studies suggested that the $\mathrm{P} 1$ component was affected by the gaze direction. ${ }^{33,34}$ The difference may happen because of the difference in experimental design, where the facial emotions without gaze cue were used as their stimuli.

The main effects of gaze cue as well as its interaction with emotional cue were significantly found in the N1 feature.
A

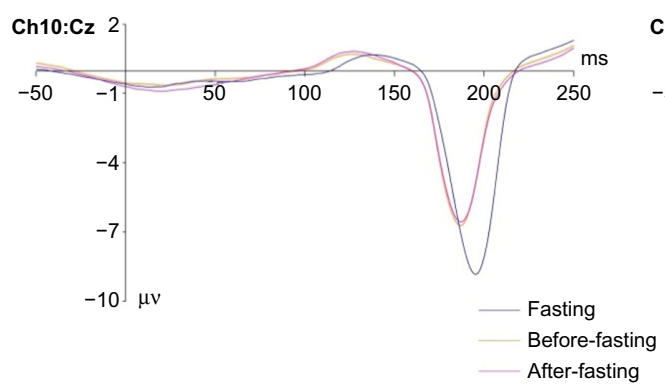

B

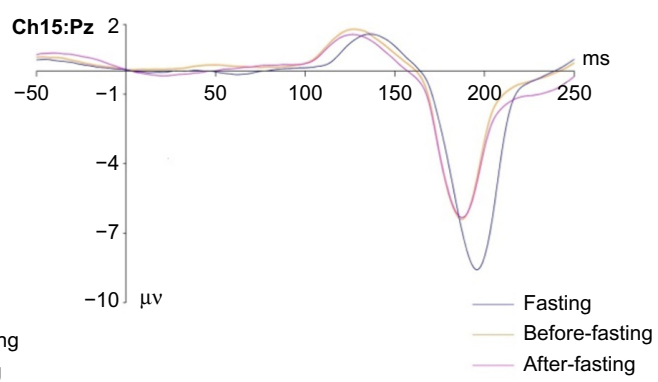

Figure 4 The significant effect of fasting on the NI component.

Notes: Bigger negative NI-amplitude during fasting period in comparison with before fasting and after fasting over middle scalp electrodes. (A) Over Cz electrode. (B) Over Pz electrode. 
A

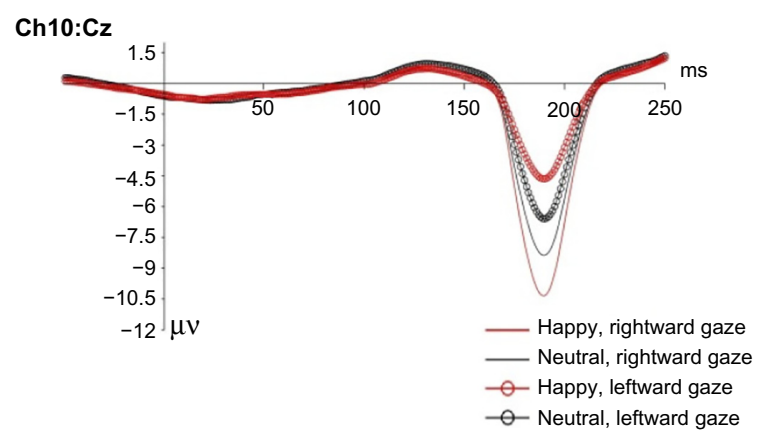

B

Ch15:Pz

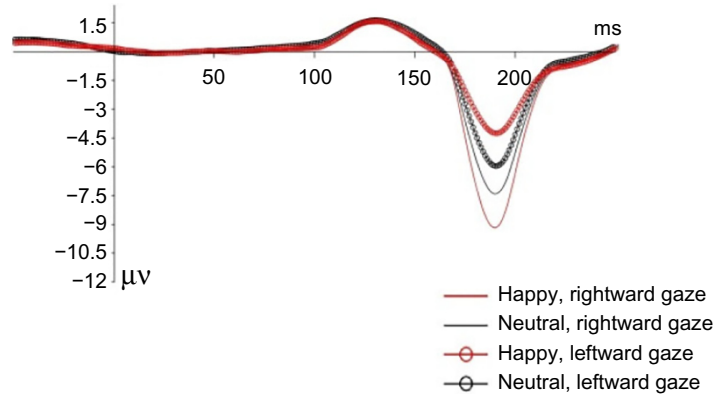

Figure 5 The effect of interaction between emotional facial stimuli and gaze cue on the NI component.

Notes: The figure illustrates that the leftward gaze cue accompanied with neutral facial stimuli induced greater NI-amplitude than that accompanied with happy face image over centroparietal electrodes. This trend was reverse for rightward gaze. The rightward gaze cue accompanied with happy face image induced greater $\mathrm{NI}$-amplitude than that accompanied with neutral facial stimuli over both electrodes. (A) Represented this trend over Cz electrode. (B) Illustrated the same trend over Pz electrode.

This finding suggested that under the processing of emotional content, the gaze cue was processed at the later stage after the processing of emotional cue. To be more detailed on the temporal dynamics of gazing and facial emotion processing, three temporal ERP stages were observed. The first stage, as well as the initial stage of early temporal visual processing, was represented by the greater $\mathrm{P} 1$-amplitude for happy facial image stimuli, and it indicated the priority of processing the emotional type during the emotional facial processing. This first-stage finding was in line with a previous study about serial processing of facial emotion. ${ }^{35}$ The second stage, which is the interaction between gaze and emotional type, happened shortly after the first stage, indicated by the changing of N1-amplitude depending on the emotional cue and the gaze cue. The third stage, which was strongly related with the changing of $\mathrm{P} 3$-amplitude, indicated the interaction process between gaze cue and target position, where the $\mathrm{P} 3$-amplitude was greater if gaze cue and target position were incongruent rather than if they were congruent. These findings were in line with a previous ERP study in dynamic emotional cue-target paradigm, which described the facial emotion and gazed eye modulated orienting of attention through the independent temporal period of processing. ${ }^{11}$

\section{A}

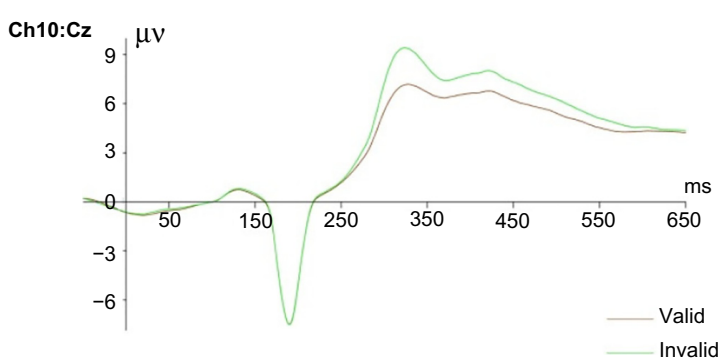

Specific to the interaction of emotional cue with gaze cue in N1-amplitude, the result observed one interesting phenomenon, where the rightward gaze cue would induce greater N1-amplitude if it was accompanied by happy facial image stimuli; however, for leftward gaze cue, a similar phenomenon happened if it was accompanied by neutral facial image stimuli but not if it was accompanied by happy facial image stimuli. This phenomenon could be explained with the lateralized theory of emotional processing, where emotional contents are processed at the right hemisphere of the brain. Leftward gaze cue would activate more the right hemisphere to process the attentional mechanism; therefore, emotional contents would engage more areas compared with that of neutral contents. As a consequence, the leftward gaze cue accompanied by neutral facial image stimuli would induce greater N1-amplitude compared with that if accompanied by happy facial image stimuli. Similar logical thinking could be used for rightward gaze cue, where it would engage the left hemisphere; therefore, the accompaniment of emotional contents with rightward gaze cue would induce greater N1-amplitude than that if accompanied with neutral facial image stimuli.

\section{B}

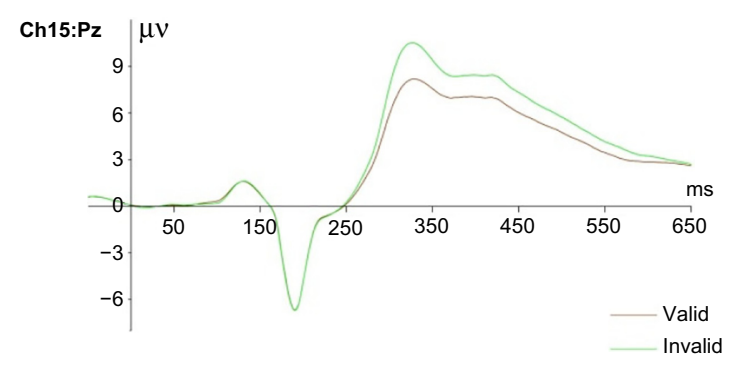

Figure 6 The validity effect on the P3 component.

Notes: The greater P3 component amplitude for invalid condition than valid condition: $(\mathbf{A})$ over the $\mathrm{Cz}$ electrode, (B) over the Pz electrode. 
Unlike lateralized theory, valence theory declared that positive emotional contents were processed in the left hemisphere of the brain..$^{12,13,36-41}$ Therefore, this phenomenon in N1-amplitude was more aligned with lateralized theory than with valence theory.

In combination of behavioral results and N1-amplitude values, this study found that fasting influenced RT, attention level, N1-amplitude, and arousal level. From the results, we observed that the value of N1-amplitude was greater during fasting than that during nonfasting. Furthermore, fasting decreased the RT, where participants tended to react slower than during nonfasting. For attention level, fasting tended to induce lower attention level compared with that during nonfasting. Arousal level was also lower during the fasting period than that during the nonfasting period. This lower arousal level might be due to the effects of fatigue and tiredness during the fasting period. Previous studies also reported the decrement of N1-amplitude due to increase of tiredness, which might make the attention level lower than it should be..$^{42,43}$ Similarly, previous studies also reported slower response from participants caused by increased tiredness. ${ }^{44,45}$

Behavioral results indicated that mood level increased during the fasting period and indicated a correlation $(r>0.8)$ with RT in response to emotional face image stimuli. This finding was aligned with the results from previous studies, which explored the effect of fasting in mood enhancement, where the positive stimuli enhanced the mood as well as attention level compared with that of neutral stimuli. ${ }^{2,3,46}$ This effect was known as mood congruency effect. ${ }^{5}$

Alteration of P3-amplitude with regard to gaze cue and target position indicated that congruency between target position and gaze cue made the RT shorter and decreased the P3-amplitude compared with that with incongruence. This decrease can be explained through contextual updating mechanisms, where faster reaction time happens because of human updated task-appropriate action to predict human future-expected event. ${ }^{47}$

\section{Conclusion}

This study utilized facial expression as a realistic social signal to explore the effects of fasting on emotional processing through the temporal and spatial dynamics of subjects' responses. During the fasting period, deficit arousal level was increased due to tiredness, and it showed significant correlation with RT. Furthermore, the mood level during the fasting period was increased, and it showed significant correlation with RT in response to happy facial image stimuli.
Three individual temporal stages were elucidated in this study. The first was the emotional type, followed by gaze cue and its interaction with emotional type (as the second stage), and lastly the interaction between target position and gaze cue (as the third stage). These stages indicated that the emotional type could be more important than gaze direction as social cue.

By using dynamic presentation of happy facial emotion as the representative of positive emotion, the results indicated that lateralized theory is more supported by the results compared with valence theory. ERP-functional magnetic resonance imaging studies may be appropriate for future research in order to elaborate more accurate spatial resolution of brain activities during fasting and its effect on nonverbal social communication.

\section{Author contributions}

Maziyar Molavi, Jasmy Yunus, and Nugraha P Utama conceived and designed the experiments. Maziyar Molavi and Nugraha P Utama performed the experiments and analyzed the data. Maziyar Molavi, Jasmy Yunus, and Nugraha P Utama contributed reagents/materials/analysis tools. Maziyar Molavi and Nugraha P Utama wrote the paper. All authors contributed toward data analysis, drafting and revising the paper and agree to be accountable for all aspects of the work.

\section{Disclosure}

This study was partially supported by GUP-UTM grant under reference number of PY/2014/02172 and PY/2014/02364 for NPU. The authors report no other conflicts of interest in this work.

\section{References}

1. Fond G, Macgregor A, Leboyer M, Michalsen A. Fasting in mood disorders: neurobiology and effectiveness. A review of the literature. Psychiatry Res. 2013;209(3):253-258.

2. Michalsen A. Prolonged fasting as a method of mood enhancement in chronic pain syndromes: a review of clinical evidence and mechanisms Curr Pain Headache Rep. 2010;14(2):80-87.

3. Farooq S, Nazar Z, Akhtar J, et al. Effect of fasting during Ramadan on serum lithium level and mental state in bipolar affective disorder Int Clin Psychopharmacol. 2010;25(6):323-327.

4. Wadlinger HA, Isaacowitz DM. Positive mood broadens visual attention to positive stimuli. Motiv Emot. 2006;30(1):87-99.

5. Jun J, Xue-Fei C, An-Tao C. The effect of positive mood on visual attention and its mechanism. Adv Psychol Sci. 2011;19(5):701-711.

6. Ochsner KK, Kosslyn SM. The Oxford Handbook of Cognitive Neuroscience, Volume 2: The Cutting Edges (Oxford Library of Psychology). Oxford, UK: Oxford University Press; 2014.

7. Putman P, Hermans E, van Honk J. Anxiety meets fear in perception of dynamic expressive gaze. Emotion. 2006;6(1):94-102.

8. Hori E, Tazumi T, Umeno K, et al. Effects of facial expression on shared attention mechanisms. Physiol Behav. 2005;84(3):397-405. 
9. Fichtenholtz HM, Hopfinger JB, Graham R, Detwiler JM, LaBar KS. Happy and fearful emotion in cues and targets modulate event-related potential indices of gaze-directed attentional orienting. Soc Cogn Affect Neurosci. 2007;2(4):323-333.

10. Graham R, Friesen CK, Fichtenholtz HM, LaBar KS. Modulation of reflexive orienting to gaze direction by facial expressions. Vis Cogn. 2010;18(3):331-368.

11. Fichtenholtz HM, Hopfinger JB, Graham R, Detwiler JM, LaBar KS. Event-related potentials reveal temporal staging of dynamic facial expression and gaze shift effects on attentional orienting. Soc Neurosci. 2009;4(4):317-331.

12. Lassalle A, Itier RJ. Fearful, surprised, happy, and angry facial expressions modulate gaze-oriented attention: behavioral and ERP evidence. Soc Neurosci. 2013;8(6):583-600.

13. Fusar-Poli P, Placentino A, Carletti F, et al. Laterality effect on emotional faces processing: ALE meta-analysis of evidence. Neurosci Lett. 2009;452(3):262-267.

14. Alpers GW. Eye-catching: right hemisphere attentional bias for emotional pictures. Laterality. 2008;13(2):158-178.

15. Alves NT, Aznar-Casanova JA, Fukusima SS. Patterns of brain asymmetry in the perception of positive and negative facial expressions. Laterality. 2009;14(3):256-272.

16. Killgore WD, Yurgelun-Todd DA. The right-hemisphere and valence Affect Neurosci. 2007;2(3):240-250.

17. Voyer D, Voyer SD, Tramonte L. Free-viewing laterality tasks: a multilevel meta-analysis. Neuropsychology. 2012;26(5):551-567.

18. Onal-Hartmann C, Pauli P, Ocklenburg S, Gunturkun O. The motor side of emotions: investigating the relationship between hemispheres, motor reactions and emotional stimuli. Psychol Res. 2012;76(3):311-316.

19. Shobe ER. Independent and collaborative contributions of the cerebral hemispheres to emotional processing. Front Hum Neurosci. 2014;8:230.

20. Lindell AK. Continuities in emotion lateralization in human and non-human primates. Front Hum Neurosci. 2013;7:464

21. Friesen C, Kingstone A. The eyes have it! Reflexive orienting is triggered by nonpredictive gaze. Psychon Bull Rev. 1998;5(3):490-495.

22. Langner O, Dotsch R, Bijlstra G, Wigboldus DH, Hawk ST, van Knippenberg A. Presentation and validation of the Radboud Faces Database. Cogn Emotion. 2010;24(8):1377-1388.

23. LaBar KS, Crupain MJ, Voyvodic JT, McCarthy G. Dynamic perception of facial affect and identity in the human brain. Cereb Cortex. 2003;13(10):1023-1033.

24. Busse L, Woldorff MG. The ERP omitted stimulus response to "no-stim" events and its implications for fast-rate event-related fMRI designs. Neuroimage. 2003;18(4):856-864.

25. Becker MW, Leinenger M. Attentional selection is biased toward mood-congruent stimuli. Emotion. 2011;11(5):1248-1254.

26. O'Haire HE. The influence of gaze direction on approach- vs avoidanceoriented emotions. Student Pulse. 2011;3(03):1. Available from: http:// www.studentpulse.com/a?id=419. Accessed February 2, 2016.

27. Bayliss AP, Schuch S, Tipper SP. Gaze cueing elicited by emotional faces is influenced by affective context. Vis Cogn. 2010;18(8):1214-1232.

28. Pecchinenda A, Pes M, Ferlazzo F, Zoccolotti P. The combined effect of gaze direction and facial expression on cueing spatial attention. Emotion. 2008;8(5):628-634. hypotheses: could they both be right (and sometimes left)? Soc Cogn

29. Bayless SJ, Glover M, Taylor MJ, Itier RJ. Is it in the eyes? Dissociating the role of emotion and perceptual features of emotionally expressive faces in modulating orienting to eye gaze. Vis Cogn. 2011;19(4):483-510.

30. Vanlessen N, Rossi V, De Raedt R, Pourtois G. Positive emotion broadens attention focus through decreased position-specific spatial encoding in early visual cortex: evidence from ERPs. Cogn Affect Behav Neurosci. 2013;13(1):60-79.

31. Di Russo F, Pitzalis S. EEG-fMRI combination for the study of visual perception and spatial attention. In: Mangun GR, editor. Cognitive Electrophysiology of Attention. San Diego, CA: Academic Press; 2014:58-70.

32. Nelson AJ, Adams RB, Stevenson MT, Weisbuch M, Norton MI. Approach-avoidance movement influences the decoding of anger and fear expressions. Soc Cogn. 2013;31(6):745-757.

33. Schuller AM, Rossion B. Perception of static eye gaze direction facilitates subsequent early visual processing. Clin Neurophysiol. 2004;115(5):1161-1168.

34. Schuller AM, Rossion B. Spatial attention triggered by eye gaze enhances and speeds up visual processing in upper and lower visual fields beyond early striate visual processing. Clin Neurophysiol. 2005;116(11):2565-2576.

35. Utama NP, Takemoto A, Koike Y, Nakamura K. Phased processing of facial emotion: an ERP study. Neurosci Res. 2009;64(1):30-40.

36. Prete G, Laeng B, Fabri M, Foschi N, Tommasi L. Right hemisphere or valence hypothesis, or both? The processing of hybrid faces in the intact and callosotomized brain. Neuropsychologia. 2015;68:94-106.

37. Nakamura A, Maess B, Knosche TR, Friederici AD. Different hemispheric roles in recognition of happy expressions. PLoS One. 2014;9(2):e88628.

38. Thomas NA, Wignall SJ, Loetscher T, Nicholls ME. Searching the expressive face: evidence for both the right hemisphere and valencespecific hypotheses. Emotion. 2014;14(5):962-977.

39. Baijal S, Srinivasan N. Emotional and hemispheric asymmetries in shifts of attention: an ERP study. Cogn Emotion. 2011;25(2):280-294.

40. Jansari A, Rodway P, Goncalves S. Identifying facial emotions: valence specific effects and an exploration of the effects of viewer gender. Brain Cogn. 2011;76(3):415-423.

41. Dal Monte O, Krueger F, Solomon JM, et al. A voxel-based lesion study on facial emotion recognition after penetrating brain injury. Soc Cogn Affect Neurosci. 2013;8(6):632-639.

42. Sambeth A, Maes JH, Quian Quiroga R, Coenen AM. Effects of stimulus repetitions on the event-related potential of humans and rats. Int J Psychophysiol. 2004;53(3):197-205.

43. Boksem MA, Meijman TF, Lorist MM. Effects of mental fatigue on attention: an ERP study. Brain Res Cogn Brain Res. 2005;25(1):107-116.

44. Sabzi AH. The effect of different fatigue protocols on choice reaction time. Middle-East J Sci Res. 2012;12:1092-1096.

45. Özdemir RA, Kirazcı S, Uğraş A. Simple reaction time and decision making performance after different physical workloads: an examination with elite athletes. Int J Hum Sci. 2010;7(2):655-670.

46. Mousavi SA, Rezaei M, Amiri Baghni S, Seifi M. Effect of fasting on mental health in the general population of Kermanshah, Iran. J Fasting Health. 2014;2(2):65-70.

47. Geng JJ, Vossel S. Re-evaluating the role of TPJ in attentional control: contextual updating? Neurosci Biobehav Rev. 2013;37(10 Part 2): $2608-2620$.

Psychology Research and Behavior Management

\section{Publish your work in this journal}

Psychology Research and Behavior Management is an international, peerreviewed, open access journal focusing on the science of psychology and its application in behavior management to develop improved outcomes in the clinical, educational, sports and business arenas. Specific topics covered include: Neuroscience, memory \& decision making; Behavior Submit your manuscript here: http://www.dovepress.com/psychology-research-and-behavior-management-journal

\section{Dovepress}

modification \& management; Clinical applications; Business \& sports performance management; Social and developmental studies; Animal studies. The manuscript management system is completely online and includes a quick and fair peer-review system. Visit http://www.dovepress. com/testimonials.php to read real quotes from published authors. 